\title{
Faster and Simpler Minimal Conflicting Set Identification*
}

\author{
Aida Ouangraoua ${ }^{\dagger} \quad$ Mathieu Raffinot R $^{\ddagger}$
}

April 4, 2019

\begin{abstract}
Let $\mathcal{C}$ be a finite set of $n$ elements and $\mathcal{R}=\left\{r_{1}, r_{2}, \ldots, r_{m}\right\}$ a family of $m$ subsets of $\mathcal{C}$. A subset $\mathcal{X}$ of $\mathcal{R}$ verifies the Consecutive Ones Property $(C 1 P)$ if there exists a permutation $P$ of $\mathcal{C}$ such that each $r_{i}$ in $\mathcal{X}$ is an interval of $P$. A Minimal Conflicting Set (MCS) $\mathcal{S} \subseteq \mathcal{R}$ is a subset of $\mathcal{R}$ that does not verify the $\mathrm{C} 1 \mathrm{P}$, but such that any of its proper subsets does. In this paper, we present a new simpler and faster algorithm to decide if a given element $r \in \mathcal{R}$ belongs to at least one MCS. Our algorithm runs in $O\left(n^{2} m^{2}+n m^{7}\right)$, largely improving the current $O\left(m^{6} n^{5}(m+n)^{2} \log (m+n)\right)$ fastest algorithm of [Blin et al, CSR 2011]. The new algorithm is based on an alternative approach considering minimal forbidden induced subgraphs of interval graphs instead of Tucker matrices.
\end{abstract}

\section{Introduction}

Let $\mathcal{C}=\left\{c_{1}, \ldots, c_{n}\right\}$ be a finite set of $n$ elements and $\mathcal{R}=\left\{r_{1}, r_{2}, \ldots, r_{m}\right\}$ a family of $m$ subsets of $\mathcal{C}$. Those sets can be seen as a $m \times n$-1 matrix $M=(\mathcal{R}, \mathcal{C})$, such that the set $\mathcal{C}$ represents the columns of the matrix, and the set $\mathcal{R}$ the rows of the matrix: each $r_{i} \in \mathcal{R}$ represents the set of columns where row $i$ has an entry 1 .

A subset $\mathcal{X}$ of $\mathcal{R}$ verifies the consecutive ones property (C1P) if there exists a permutation $P$ of $\mathcal{C}$ such that each $r_{i}$ in $\mathcal{X}$ is an interval of $P$. Testing the consecutive ones property is the core of many algorithms that have applications in a wide range of domains, from VLSI circuit conception through planar embeddings [8] to computational biology for the reconstruction of ancestral genomes [1, 2, 4, 5, 9. We focus on this last field in this paper.

On real biological matrices, the $\mathrm{C} 1 \mathrm{P}$ is rarely verified, and only some subsets of rows might verify the desired property.However, the combinatorics of such sets is difficult to handle, and a strategy to deal with them has been proposed in [1, 5, 9]. It consists in identifying the rows belonging to minimal conflicting subsets of rows that do not verify the $\mathrm{C} 1 \mathrm{P}$, but such that any of their row subset does.

\footnotetext{
*This work is partly supported by the french MAPPI project (ANR-2010-COSI-004).

${ }^{\dagger}$ INRIA, Centre de recherche INRIA Haute-Borne, Bât. A, Park Plaza 40 avenue Halley, 59650 Villeneuve d'Ascq, France. aida.ouangraoua@inria.fr

${ }^{\ddagger}$ CNRS/LIAFA, Université Paris Diderot - Paris 7, France, raffinot@liafa.jussieu.fr
} 
Definition 1 A set $\mathcal{S} \subseteq \mathcal{R}, \mathcal{S} \neq \emptyset$ is a Minimal Conflicting Set (MCS) if $\mathcal{S}$ does not verify the $C 1 P$, but such that $\forall \mathcal{X}, \mathcal{X} \subset S$, the set $\mathcal{X}$ verifies the $C 1 P$.

However, it is not difficult to build examples of matrices such that the number of MCS is polynomial or even exponential in the number of rows.

Figure 1 shows such an example in which each sub set of 3 rows is a MCS. Thus, such a construction with $m$ rows gives $C_{3}^{m}=O\left(m^{3}\right)$ MCS. Note that, on this example, a single row is included in $O\left(m^{2}\right)$ MCS.

Figure 2,(a) shows another example where the number of MCS is exponential in the number of rows. Let $k$ be the number of nodes of external rows, which are $r_{7}, r_{8}$, and $r_{9}$ on the figure. The total number of rows is

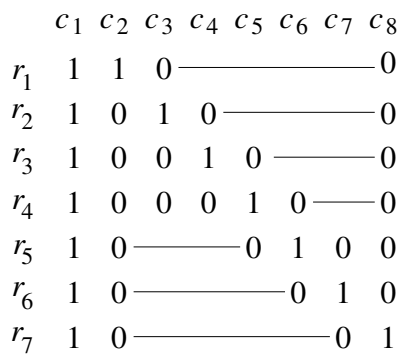

Figure 1: A matrix not verifying the $\mathrm{C} 1 \mathrm{P}$ and such that each set of 3 rows is a MCS. $3 k$, the number of columns $2 k$, and the number of MCS is $2^{k}$ since any induced chordless cycle in the row intersection graph of the matrix (Figure2-(b)) constitutes a MCS.

\begin{tabular}{cccccccc} 
& \multicolumn{1}{c}{$\mathbf{c}_{\mathbf{1}}$} & $c_{2}$ & $\mathbf{c}_{3}$ & $c_{4}$ & $\mathbf{c}_{5}$ & $c_{6}$ \\
$r_{1}$ & 1 & 0 & 0 & 0 & 0 & 1 \\
$r_{2}$ & 1 & 1 & 0 & 0 & 0 & 0 \\
$r_{3}$ & 0 & 1 & 1 & 0 & 0 & 0 \\
& $r_{4}$ & 0 & 0 & 1 & 1 & 0 & 0 \\
(a) & $r_{5}$ & 0 & 0 & 0 & 1 & 1 & 0 \\
& $r_{6}$ & 0 & 0 & 0 & 0 & 1 & 1 \\
$r_{7}$ & 1 & 1 & 0 & 0 & 0 & 0 \\
$r_{8}$ & 0 & 0 & 1 & 1 & 0 & 0 \\
$r_{9}$ & 0 & 0 & 0 & 0 & 1 & 1
\end{tabular}

(b)

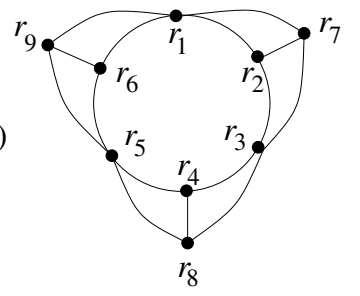

Figure 2: (a) A matrix not verifying the $\mathrm{C} 1 \mathrm{P}$ and such that the number of MCS is exponential in the number of rows. (b) A row intersection graph of the matrix whose vertices correspond to the rows of the matrix, and such that there exists an edge between two rows $r_{i}$ and $r_{j}$ if $r_{i} \cap r_{j} \neq \emptyset$.

From a computational point of view, the first question that arises is the following: is a given row $r \in \mathcal{R}$ included in at least one MCS ? This question has been raised in [1, recalled in [4, 5] and recently solved in polynomial time $O\left(m^{6} n^{5}(m+n)^{2} \log (m+n)\right)$ in 3 . This currently fastest algorithm is based on the identification of minimal Tucker forbidden submatrices [10, 6].

In this paper we present a new simpler $O\left(m^{2} n^{2}+n m^{7}\right)$ time algorithm for deciding if a given row belongs to at least one MCS and if true exhibit one. Our algorithm is based on an alternative approach considering minimal forbidden 
induced subgraphs of interval graphs [7] instead of Tucker matrices. Moreover, our central paradigm consists in reducing the recognition of complex forbidden induced subgraphs to the detection of induced cycles in ad-hoc graphs, while in [3. only induced paths are considered. Our approach is faster and simpler, but a limit shared by both approaches resides in avoiding to report the number of MCS to which a given row belongs.

\section{MCS and Forbidden induced subgraphs}

The row-column intersection graph of a $0-1$ matrix $M=(\mathcal{R}, \mathcal{C})$ is a vertexcolored bipartite graph $G_{R C}(M)$ whose set of vertices is $\mathcal{R} \cup \mathcal{C}$; the vertices corresponding to rows (resp. columns) are black (resp. white) ; there exists an edge between two rows $r_{i} \in \mathcal{R}$ and $r_{j} \in \mathcal{R}$ if $r_{i} \cap r_{j} \neq \emptyset$, and there exists an edge between a row $r \in \mathcal{R}$ and a column $c \in \mathcal{C}$ if $c \in r$.

It should be noted that a column vertex (white) is only connected to row vertices (black).

The neighborhood $N(r)$ of a row $r$ is the set of rows intersecting $r, N(r)=$ $\{x \in \mathcal{R}: r \cap x \neq \emptyset\}$ and $N\left(r_{i}, r_{j}\right)=N\left(r_{i}\right) \cap N\left(r_{j}\right)$. The span $L(c)$ of a column $c$ is the set of rows containing $x, L(c)=\{r \in \mathcal{R}: c \in r\}$.
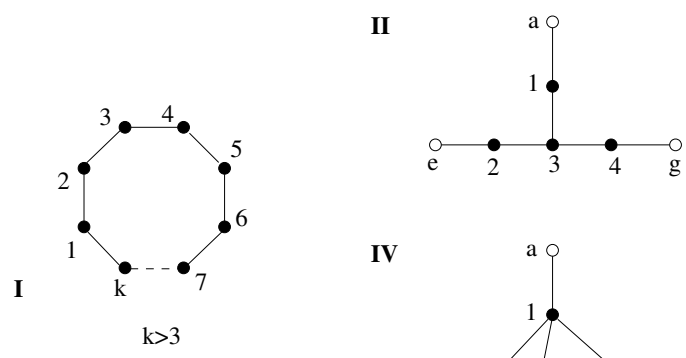

IV

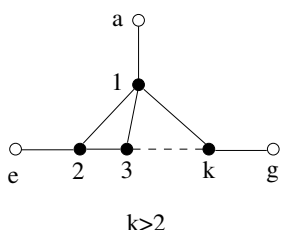

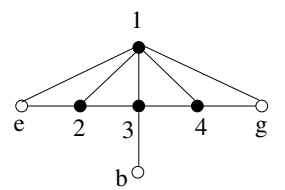

III

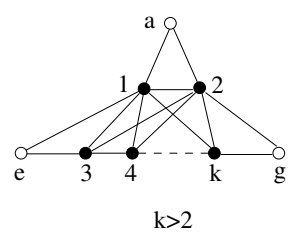

Figure 3: Forbidden induced subgraphs for the row-column intersection graph of $M=(\mathcal{R}, \mathcal{C})$ to verify $\mathrm{C} 1 \mathrm{P}$.

Theorem 1 ([7], Theorem 4) A 0 -1 matrix $M=(\mathcal{R}, \mathcal{C})$ verifies the $C 1 P$ if and only if its row-column intersection graph does not contain a forbidden induced subgraph of the form I, II, III, IV, or V (Figure 3).

Property 1 From Theorem 1, a set $\mathcal{S} \subseteq \mathcal{R}$ is a MCS if the row-column intersection graph $G_{R C}(\mathcal{S}, \mathcal{C})$ contains a subgraph of the form I, II, III, IV, or $V$; and for any $\mathcal{T} \subset \mathcal{S}, G_{R C}(\mathcal{T}, \mathcal{C})$ does not contain a subgraph of the form I, II, III, IV, or $V$. 
Given a MCS $\mathcal{S} \subseteq \mathcal{R}$, a forbidden induced subgraph contained in $G_{R C}(\mathcal{S}, \mathcal{C})$ is said to be responsible for the MCS $\mathcal{S}$. If this forbidden induced subgraph is of the form I (resp. II; III; IV; V), we simply say that $\mathcal{S}$ is a MCS of the form I (resp. II; III; IV; V).

Definition 2 A row of a MCS $\mathcal{S}$ that intersects all other rows of $\mathcal{S}$ is called a kernel of $\mathcal{S}$. In a forbidden induced subgraph responsible for $\mathcal{S}$, any kernel of $\mathcal{S}$ constitutes a black vertex that is connected to all other black vertices.

Property 2 Note that an induced subgraph of the form II, III, IV, or V necessarily contains at least one kernel, while an induced subgraph of the form I contains no kernel.

We denote by $G_{R}(M)$, the subgraph of $G_{R C}(M)$ induced by the set of rows $\mathcal{R}$, thus containing only black vertices.

Graph sizes. $G_{R}(M)$ has $m$ vertices and at $\operatorname{most} \min \left(m n, m^{2}\right)$ edges, while $G_{R C}(M)$ has $m+n$ vertices and at most $\min \left((m+n)^{2}, m^{2} n\right)$ edges.

\section{A global algorithm}

Our algorithm to decide if a row $r \in \mathcal{R}$ of a 0 -1 matrix $M=(\mathcal{R}, \mathcal{C})$ belongs to at least one MCS, is based on a sequence of algorithms for finding a forbidden subgraph of $G_{R C}(M)$ responsible for a MCS containing $r$. It looks for forbidden subgraph of the form I, III, II, IV, V, in the following order: 1. MCS of type I, 2. MCS of size 3 (types IV or V), 3. MCS of type II, 4. MCS of type III, 5. MCS of type IV and size larger or equal to 4, and MCS of type V and size larger or equal to 4 . See Figure 4 for an overview. The steps 1 to 4 are based on straightforward brute-force algorithms, while the two last steps relies to a reduction to the detection of induced chordless cycles in ad-hoc graphs.

In the following, we simply write $G_{R C}(M)$ as $G$ and $G_{R}(M)$ as $G_{R}$.

\subsection{Step 1: Forbidden induced subgraph I}

We first test if $r$ belongs to a MCS of the form I. If it is true, then $r$ belongs to an induced chordless cycle of $G$ of length at least 4 containing only black vertices. Such a cycle exists in $G$ if and only if is also a chordless cycle in $G_{R}$ since $G_{R}$ is the subgraph of $G$ induced by the set of rows $\mathcal{R}$. Thus it suffices to search for an induced chordless cycle in $G_{R}$.

Proposition 1 Algorithm Check_I is correct and runs in worst case $O\left(m^{5}\right)$ time.

Proof. The correctness of Algorithm Check_I comes from the fact that, $r$ is contained in a MCS of the form I if and only if $r$ belongs to an induced chordless cycle of $G_{R}$ of length at least 4 whose set of vertices $\mathcal{S}$ constitutes the MCS (Figure 4.I). A $P_{4}$ of $G_{R}$ is an induced chordless path of $G_{R}$ containing 4 vertices. 


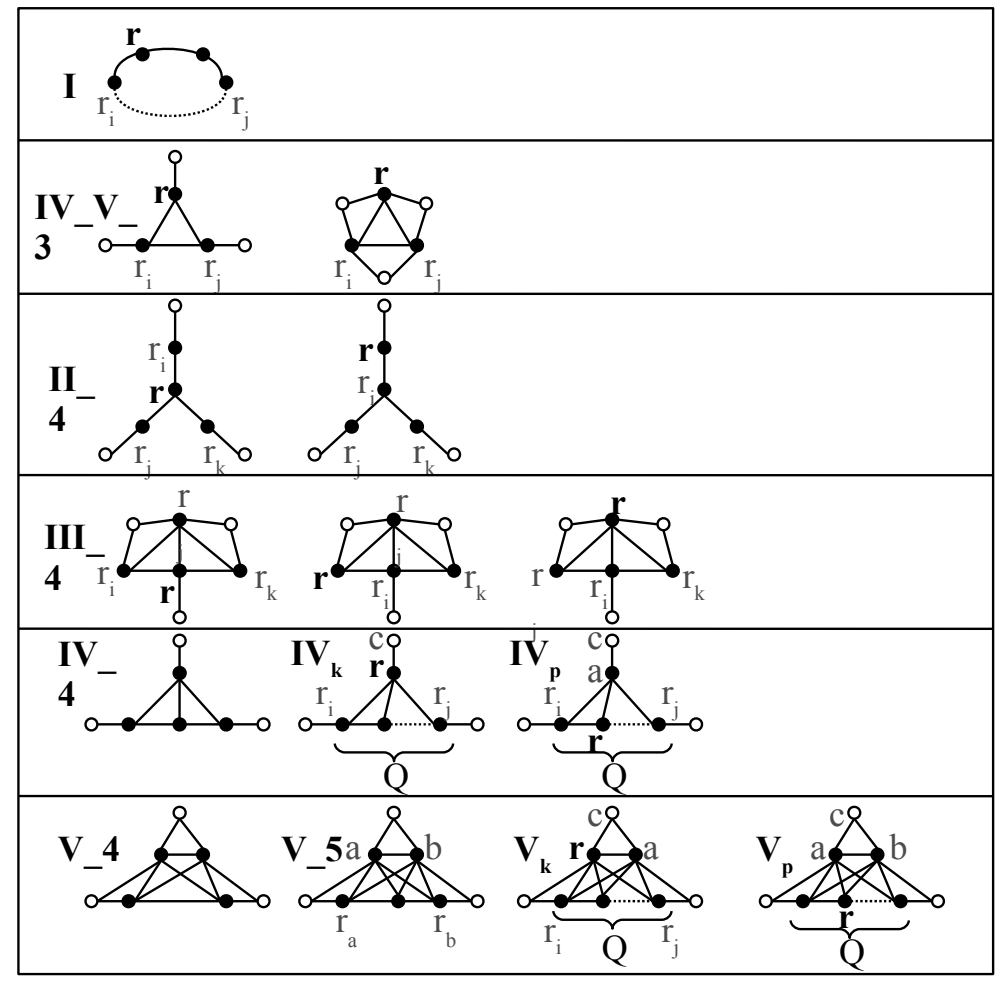

Figure 4: The different steps of the algorithm: in each case, when row $r$ has a specific location in the forbidden induced subgraph that is looked for, this location is indicated in bold character. Other rows and columns of the forbidden induced subgraph are indicated in grey color characters.

In this case, Algorithm Check_I returns such a set of vertices since an induced chordless cycle of $G_{R}$ of length at least 4 containing $r$ is a $P_{4}$ containing $r$ whose extremities are linked by a chordless path in the subgraph of $G$ that does not contain the neighborhood of the internal vertices of the $P_{4}$. This set $\mathcal{S}$ cannot contain a smaller subset of rows that is a MCS, as no subset of $S$ can be a MCS of the form I, or a MCS of any other form because of Property 2 .

Algorithm Check_I might be implemented in $O\left(m^{5}\right)$. The test performed on a give $P_{4}$ containing $r$ (lines 2-5 of the algorithm) can be achieved in $O\left(\min \left(m n, m^{2}\right)+m \log m\right)$ as follows: removing the neighborhood of its internal vertices might be done in $\min \left(m n, m^{2}\right)$ time, and finding a chordless path between the two extremities might be performed using Dijkstra's algorithm in $O\left(\min \left(m n, m^{2}\right)+m \log m\right)$ time. Enumerating all $P_{4}$ containing $r$ might be done in time $O\left(\mathrm{~m}^{3}\right)$ using a BFS from $r$ stopping at depth 4. Eventually, the whole algorithm is in $O\left(m^{3}\left(\min \left(m n, m^{2}\right)+m \log m\right)\right)=O\left(m^{5}\right)$ time. 


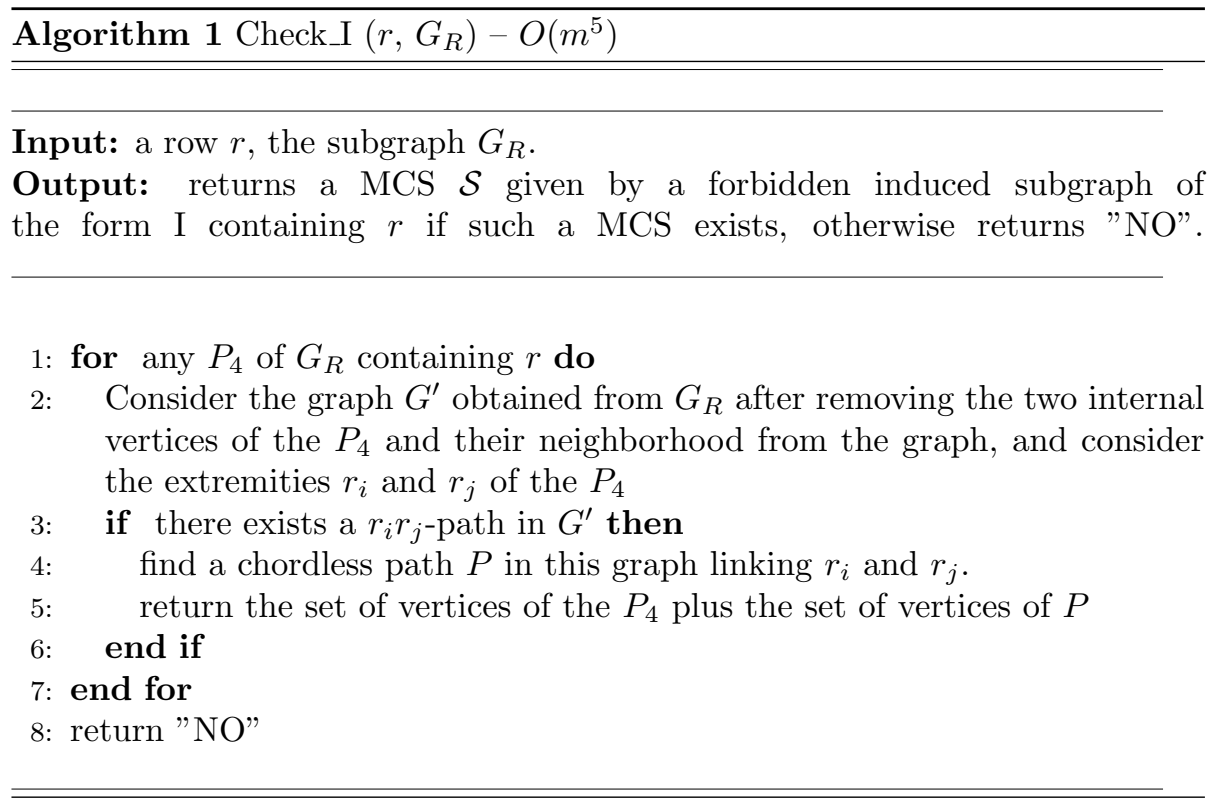

Precomputation. In the following steps, we assume that the following precomputations have been achieved:

- For any triplet of rows $\left(r, r_{i}, r_{j}\right)$ that are pairwise intersecting, i.e each couple is an edge in $G, r-\left(r_{i} \cup r_{j}\right)$ and $\left(r_{i} \cap r_{j}\right)-r$ are precomputed ;

- Two rows $r_{i}$ and $r_{j}$ are overlapping if $r_{i} \cap r_{j} \neq \emptyset$ and $r_{i}-r_{j} \neq \emptyset$ and $r_{j}-r_{i} \neq \emptyset$. The overlapping relation between any couple of rows is precomputed ;

- For any quadruplet of rows $\left(r, r_{i}, r_{j}, r_{k}\right)$ such that $r_{i}, r_{j}$, and $r_{k}$ overlap $r$, $r-\left(r_{i} \cap r_{j} \cap r_{k}\right)$ is precomputed.

All those precomputations can simply be performed in $O\left(m^{4} n\right)$ time using straightforward algorithms, that is, scanning the $n$ columns of the input matrix for each triplet or quadruplet of rows.

\subsection{Step 2: Forbidden induced subgraph responsible for a MCS of size 3}

We test here if $r$ belongs to a MCS of size 3. A MCS of size 3 is necessarily caused by a forbidden induced subgraph of the form IV or V. As a consequence, the following property is immediate.

Property 3 A MCS of size 3 is always composed of 3 rows that are pairwise overlapping. 


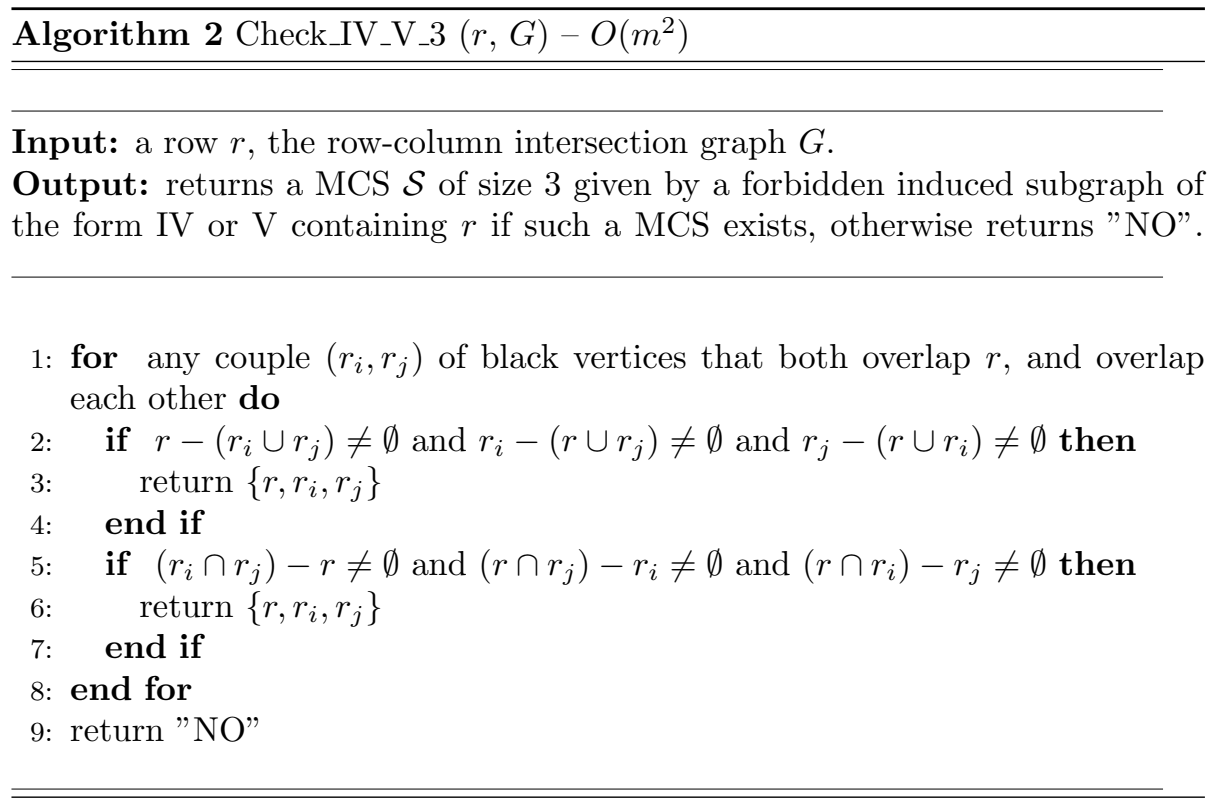

Proposition 2 Algorithm Check $I_{-} V_{-} V_{-} 3$ is correct and runs in $O\left(m^{2}\right)$ time.

Proof. The correctness of Algorithm Check_IV_V_3 comes from the fact that, $r$ is contained in a MCS of size 3 if and only if this MCS is caused by a forbidden induced subgraph of the form IV or V (Property 3). Thus, $r$ should belong to a triplet of rows $\left(r, r_{i}, r_{j}\right)$ that are pairwise overlapping, and satisfy the conditions given in:

- either, line 2 of the algorithm to produce a forbidden induced subgraph of the form IV (left-end graph in Figure 4 IV_V_3),

- or, line 5 of the algorithm to produce a forbidden induced subgraph of the form V (right-end graph in Figure 4 IV_V_3).

In both cases, Algorithm Check_IV_V_3 returns the set $\left\{r, r_{i}, r_{j}\right\}$ as a MCS if such a set of rows exists. This set cannot contain a smaller subset of rows that is a MCS as 3 is the minimum size of any MCS.

Algorithm Check_IV_V_3 runs in $O\left(m^{2}\right)$ time since, given $r$, there might be $O\left(m^{2}\right)$ couples $\left(r_{i}, r_{j}\right)$ on which the tests performed (lines 2-8 of the algorithm) might be achieved in $O(1)$, thanks to the precomputations that have been done.

\subsection{Step 3: Forbidden induced subgraph II}

We test here if $r$ belongs to a MCS of the form II, with the assumption that $r$ is not contained in any MCS of size 3. Note that such a MCS is of size 4 . 


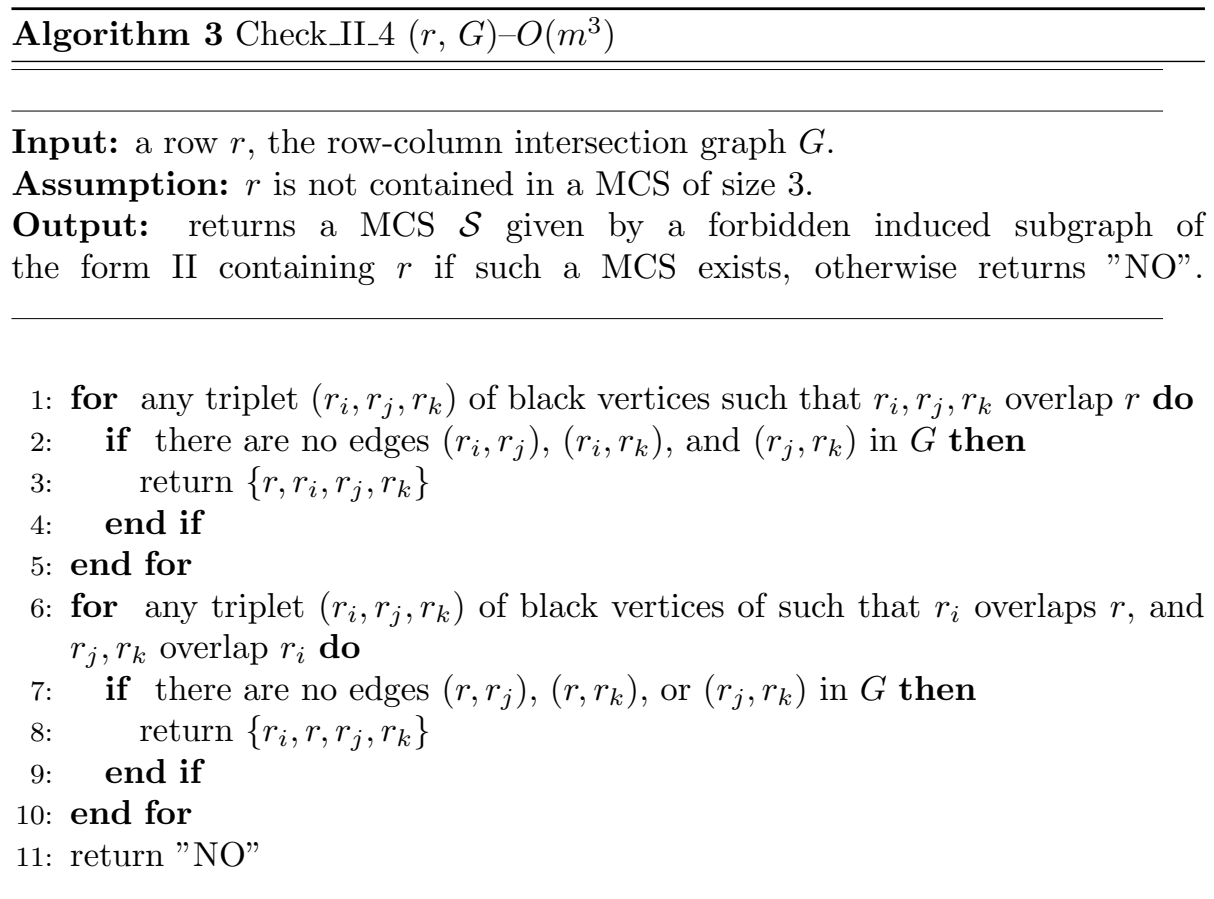

Proposition 3 Algorithm Check_II_4 is correct and runs in $O\left(m^{3}\right)$ time.

Proof. The correctness of Algorithm Check_II_4 comes from the fact that, if $r$ belongs to a MCS of the form II, then $r$ should belong to a quadruplet of rows $\left(r, r_{i}, r_{j}, r_{k}\right)$ such that one these rows is a kernel, and the three other rows do not intersect each other. Thus, the row $r$ is:

- either, a kernel of the MCS, tested in lines 1-5 of the algorithm (left-end graph in Figure 4.II_4),

- or, not a kernel of the MCS tested in lines 6-10 of the algorithm (right-end graph in Figure 4.II_4).

In both cases, Algorithm Check_II_4 returns the set $\left\{r, r_{i}, r_{j}, r_{k}\right\}$ as a MCS if such a set of rows exists. This set cannot contain a smaller subset of rows that is a MCS as this subset would be a subset of 3 rows that cannot satisfy Property 3.

Algorithm Check_IV_V_4 runs in $O\left(\mathrm{~m}^{3}\right)$ time since all the tests performed on a given triplet $\left(r_{i}, r_{j}, r_{k}\right)$ in lines 2-4 and 7-9 of algorithm can be achieved in $O(1)$, and given $r$ there might be $O\left(m^{3}\right)$ such triplets. 


\subsection{Step 4: Forbidden induced subgraph III}

We test here if $r$ belongs to a MCS of the form III, with the assumption that $r$ is not contained in a MCS of size 3. Note that such a MCS is of size 4 .

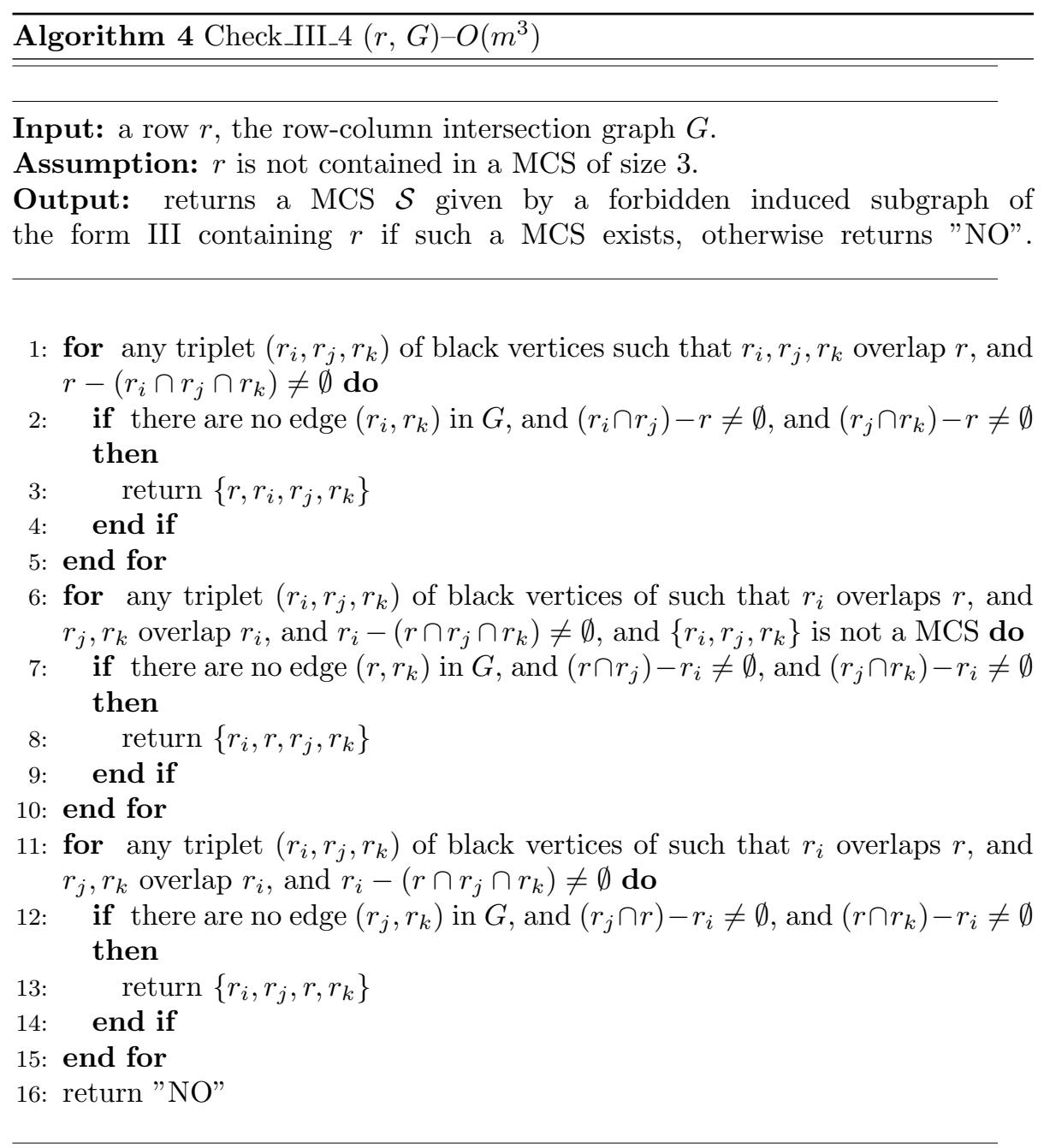

Proposition 4 Algorithm Check_III_4 is correct and runs in $O\left(m^{3}\right)$ time.

Proof. The correctness of Algorithm Check_III_4 comes from the fact that, $r$ belongs to a MCS of the form III if and only if $r$ should belong to a quadruplet of rows $\left(r, r_{i}, r_{j}, r_{k}\right)$ included in an induced subgraph of the form III such that two of these rows are kernels of the subgraph, and one of these kernels contains a column of the induced subgraph that is not shared with any of the other rows. 
Let us call this kernel kernel_1, and the other kernel kernel_2. For example in the left-end graph in Figure 4.III_4, kernel_1 $=r$, and kernel_ $2=r_{j}$.

Thus, the row $r$ is:

- either, kernel_1, tested in lines 1-5 of the algorithm (left-end graph in Figure 4.III_4),

- or, not a kernel, tested in lines 6-10 of the algorithm (middle graph in Figure 4[III_4).

- or, kernel_2, tested in lines 11-15 of the algorithm (right-end graph in Figure 4.III_4).

In the first, and third cases, the set $\left\{r_{i}, r_{j}, r_{k}\right\}$ cannot be a MCS because such a set cannot satisfy Property 3 In all cases, Algorithm Check_III_4 returns the set $\left\{r, r_{i}, r_{j}, r_{k}\right\}$ as a MCS if such a set of rows exists, and $\left\{r_{i}, r_{j}, r_{k}\right\}$ is not a MCS (in the second case). Since we made the assumption that $r$ is not contained in a MCS of size 3, there cannot exists a smaller subset of $\left\{r_{i}, r_{j}, r_{k}\right\}$ containing $r$ that is a MCS.

Algorithm Check_III_4 runs in $O\left(\mathrm{~m}^{3}\right)$ time using a similar proof as the complexity proof for Check_IV_V_4: all the tests performed by the algorithm (lines $2-4,7-9$, and 12-14 of the algoritms) on a given triplet $\left(r_{i}, r_{j}, r_{k}\right)$ are achieved in $O(1)$ thanks to the precomputations, and given $r$ there might be $O\left(m^{3}\right)$ such triplets.

\subsection{Step 5: Forbidden induced subgraph IV}

We test here if $r$ belongs to a MCS of the form IV, with the assumption that $r$ is contained, neither in a MCS of size 3, nor in a MCS of type I. Depending on whether the size of the MCS is 4 or larger than 4, we describe two algorithms.

\subsubsection{MCS of size 4}

We first test if $r$ belongs to a MCS of the form IV of size 4. We look for a triplet of rows $\left(r_{i}, r_{j}, r_{k}\right)$ such that the set $\left\{r, r_{i}, r_{j}, r_{k}\right\}$ is a MCS of the form IV (Figure 4.IV_4). In an induced subgraph of the form IV containing 4 rows $\left\{r, r_{i}, r_{j}, r_{k}\right\}$, two rows are kernels, and in that case, $r$ is either a kernel of the MCS, or not. If $r$ is a kernel, then it is either a kernel -called kernel_1- containing a column of the induced subgraph that is not shared with any of the other rows, or not -called kernel_2-. For example, in the left-end graph in Figure 4.IV_4, the two kernel are the two central black vertices of the graph: the top one is a kernel_1, and the bootom one a kernel_2. Algorithm Check_IV_4 looks for each of these configurations:

- $r$ is a kernel_1, tested in lines 1-5 of the algorithm;

- $r$ is not a kernel,tested in lines 6-10 of the algorithm;

- $r$ is a kernel $\_2$, tested in lines 11-15 of the algorithm. 
The proof of the correctness of Algorithm Check_IV_4 is similar to the proof for Algorithm Check_III_4.

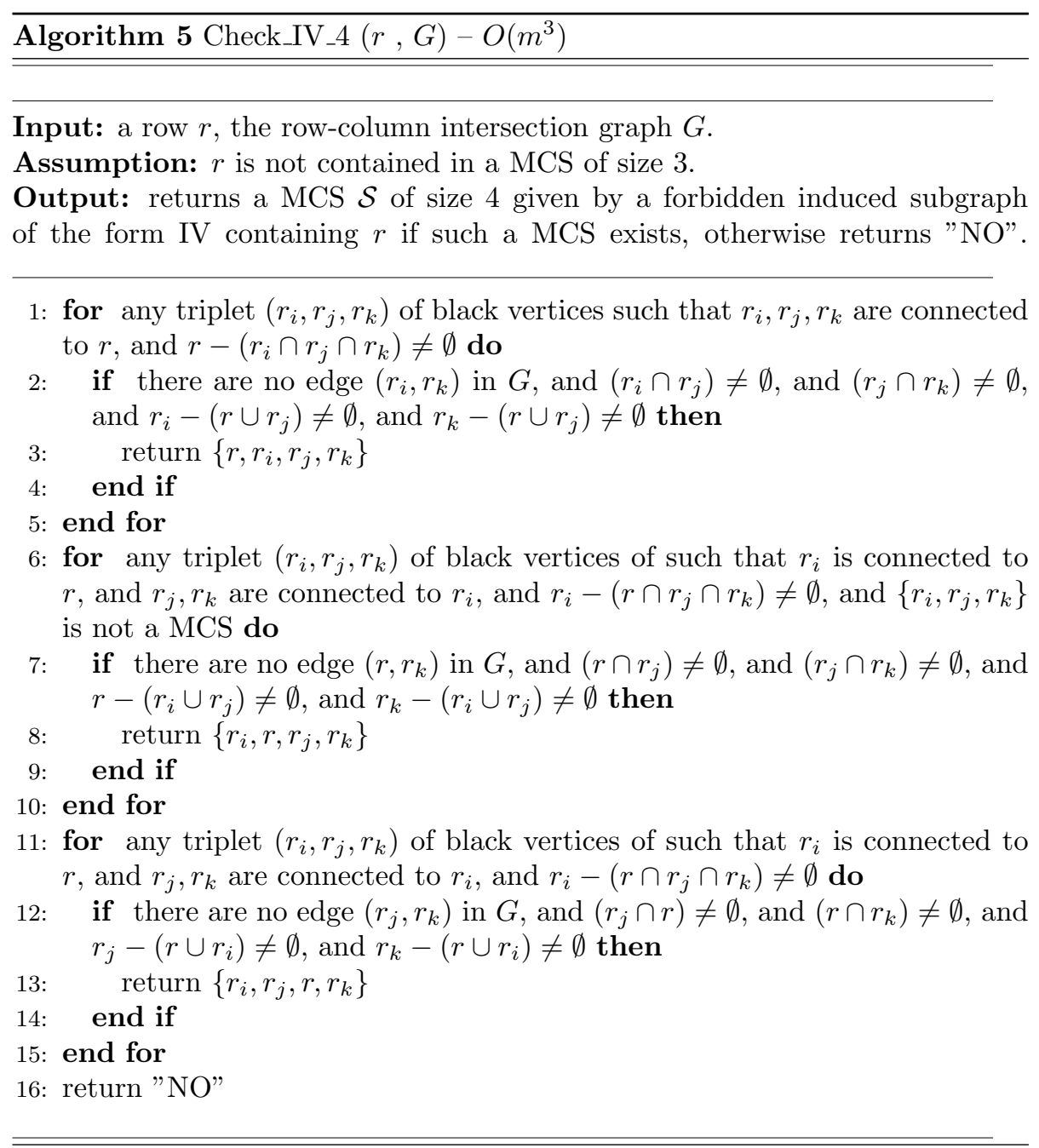

Proposition 5 Algorithm Check_IV-4 is correct and runs in $O\left(m^{3}\right)$ time.

Proof. The proof for Algorithm Check_IV_4 is similar to the proof for Algorithm Check_III_4.

\subsubsection{MCS of size larger than 4}

We test here if $r$ belongs to a MCS of the form IV of size larger than 4. A MCS of the form IV of size larger than 4 contains one and only one kernel. Depending 
on whether $r$ is the kernel or not, we distinguish two cases here.

\section{Case 1: If row $r$ is the kernel of the MCS}

Algorithm Check $I_{V}$ recovers a MCS $\mathcal{S}$ of the form IV of size larger than 4 containing $r$ as a kernel, with the assumption that $r$ is not contained in a MCS of size 3 (Figure 4 $\mathrm{IV}_{k}$ ). The principle of the algorithm relies in first choosing the column $c \in \mathcal{C}$, of the forbidden induced subgraph of type IV responsible for $\mathcal{S}$, that is contained in $r$, and in no other row of the MCS (see Figure $4 \mathrm{IV}_{k}$ ). Next, it considers the subgraph $H$ of $G$ induced by the set of black vertices (rows) that are neighbors of $r$, but do not contain the column $c$. We denote this subgraph by $H=G[N(r)-L(c)]$. Then, it looks for a set of rows $Q$, constituting a chordless path in $H$, such that $\{r\} \cup Q$ is a MCS of the form IV.

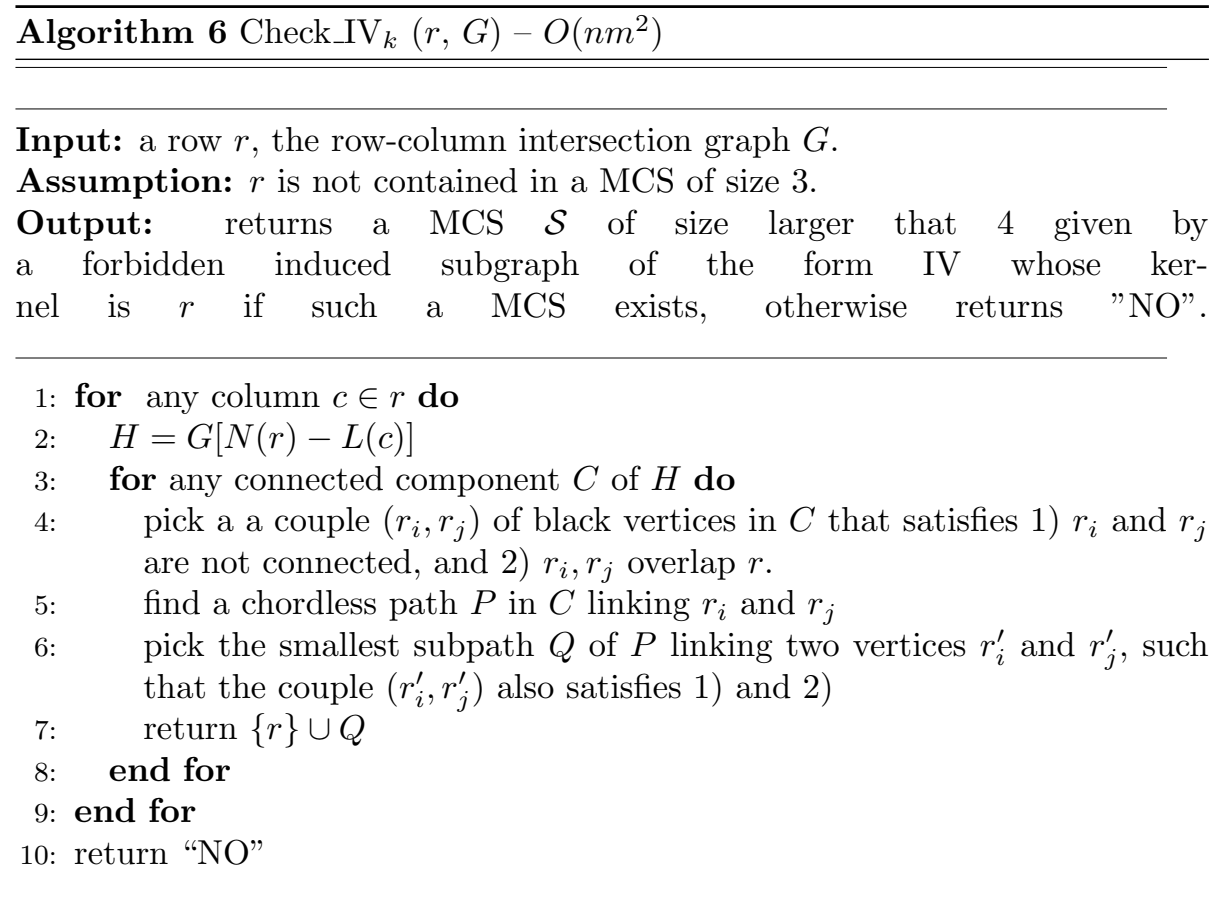

Proposition 6 Algorithm Check $k_{-} V_{k}$ is correct and runs in $O\left(\mathrm{~nm}^{2}\right)$ time.

Proof. Note that, if the MCS exists, then all the rows belonging to the MCS, except $r$, belong to a same connected component of $H$. Thus, in each connected component of $H$, the algorithm looks for a chordless path $Q$ linking two vertices $r_{i}, r_{j}$ satisfying 1) $r_{i}$ and $r_{j}$ are not connected, and 2) $r_{i}, r_{j}$ overlap $r$, and 3) $Q$ does not contain any smaller subpath satisfying conditions 1 ) and 2). These conditions are necessary and sufficient for the set $\{r\} \cup Q$ to form the rows of 
a induced subgraph of the form $I V$. The set $\{r\} \cup Q$ cannot contain a subset that is a MCS as such a smaller MCS should be:

- either a MCS of size 3 including $r$, which impossible by assumption,

- or a MCS of type II or III necessarily including $r$ as kernel,

- or a MCS of type IV and size larger than 3 having $r$ as kernel.

The two last cases are also impossible, since $Q$ would not have satisfy condition $3)$ in these cases.

Next, there might be $n$ columns $c \in r$ and up to $m^{2}$ couples $\left(r_{i}, r_{j}\right)$ of black vertices to test before finding a valid couple $\left(r_{i}, r_{j}\right)$ satisfying the conditions in line 4 of the algorithm. Up to this point, the complexity is in $O\left(\mathrm{~nm}^{2}\right)$. Assume now that such a couple exist. Then finding a chordless path between $r_{i}$ and $r_{j}$ might be done by searching for a shortest path between $r_{i}$ and $r_{j}$ in the connected component $C$ using Dijkstra's algorithm, which thus requires at worst $O\left(\min \left(m n, m^{2}\right)+m \log m\right)$ time. The path is of length at most $m$, and thus identifying $r_{i}^{\prime}$ and $r_{j}^{\prime}$ is bounded by testing each pair on this path in $C$, which requires at worst $O\left(m^{2}\right)$ time. Thus, in total, the algorithm is $O\left(n m^{2}\right)$ worst case time.

\section{Case 2: If row $r$ is not the kernel of the MCS}

Algorithm Check $I V_{p}$ recovers a MCS $\mathcal{S}$ of the form IV of size larger than 4 containing $r$, but not as a kernel, with the assumptions that $r$ is not contained in a MCS of size 3, and $r$ does not belong to an induced chordless cycle of $G_{R}$ (Figure $4 . \mathrm{IV}_{p}$ ). The principle of the algorithm consists in first choosing the kernel $a$ of $\mathcal{S}$ among the black vertices (rows) neighbors of $r$, and the column $c \in \mathcal{C}$, of the induced subgraph of type IV responsible for $\mathcal{S}$, that is contained in $a$, but in no other row of the MCS. (see Figure $4\left(\mathrm{IV}_{p}\right.$ ). Next, the algorithm calls Algorithm Check_IV to look for the MCS $\mathcal{S}$ with $r, a, c$, and $G$ given as parameters.

Algorithm Check_IV is called in Algorithm Check_IV $v_{p}$. It recovers a MCS $\mathcal{S}$ of the form IV of size larger than 4 containing $r$, given the row $r$, the kernel $a$ of the MCS $\mathcal{S}$, and the column $c \in \mathcal{C}$, of the induced subgraph of type IV responsible for $\mathcal{S}$, that is contained in $a$, but in no other row of the MCS (Figure 4. $\left.\mathrm{IV}_{p}\right)$.

Proposition 7 Algorithm Check_IV $V_{p}$ is correct, and runs in $O\left(\mathrm{~nm}^{6}\right)$ time.

Proof.

The correctness and the complexity of Check $I V_{p}$ follows directly from the the correctness and the complexity of Algorithm Check_IV that is called in Algorithm Check IV . $_{\text {. }}$

The correctness of Check_IV comes from the fact that, $r$ does not belong to any chordless cycle in the graph $C$ computed at line 2 of the algorithm by assumption. Then at line 6 of the algorithm, any chordless cycle in the graph 
$D$ containing vertex $r$ necessarily contains at least one edge $\left(r_{i}, r_{j}\right)$ belonging to the set $E_{a}$. The number of edges belonging to the set $E_{a}$ in such a chordless cycle $Q$ cannot be greater than 1 as any couple of such edges in the chordless cycle would induce a chord. Indeed, if $Q$ contains more than one edge belonging to $E_{a}$, any two such edges would have to extremities in $V_{a}$, one from each of the two edges, that are not connected in the graph $C$. These extremities would thus be linked by an edge in $E_{a}$, creating a chord for the cycle $Q$ in the graph D.

Therefore, the set of vertices of the chordless cycle $Q$ induces a chordless path in $G$ such that each vertex of $Q$ is connected to vertex $a$ by definition of the graph $H$, and the extremities $r_{i}$ and $r_{j}$ of $Q$ satisfy 1) $r_{i}$ and $r_{j}$ are not connected in $G$, and 2) $r_{i}, r_{j}$ overlap $r$, and 3) $Q$ does not contain any smaller subpath satisfying conditions 1) and 2). These conditions are necessary and sufficient for the set $\{a\} \cup Q$ to form the rows of an induced subgraph of the form $I V$, and this set cannot contain a smaller MCS since such a MCS would be:

- either a MCS of size 3 including $a$,

- or a MCS of type II or III necessarily including $a$ as kernel,

- or a MCS of type IV and size larger than 3 having $a$ as kernel.

The 3 cases are impossible, since they would induce a chord from the set $E_{a}$ in the chordless cycle induced by $Q$ in the graph $D$.

Algorithm Check_IV calls Algorithm Check_I. Both algorithms have the same time complexity in $O\left(\mathrm{~m}^{5}\right)$ time. It follows immediately that Algorithm Check_IV ${ }_{p}$ runs in $\mathrm{Onm}^{6}$ time.

\subsection{Step 6: Forbidden induced subgraph V}

We test here if $r$ belongs to a MCS of the form $\mathrm{V}$, with the assumption that $r$ is contained neither in a MCS of size 3, nor in a MCS of type I. Depending on whether the size of the MCS is 4, 5 or larger than 5 , we describe three algorithms.

\subsubsection{MCS of size 4 or 5}

We first test if $r$ belongs to a MCS of the form V of size 4 or 5 . For a MCS of size 4 , we look for a triplet of rows $\left(r_{i}, r_{j}, r_{k}\right)$ such that the set $\left(r, r_{i}, r_{j}, r_{k}\right)$ is a MCS of the form $\mathrm{V}$. In such a case, we look for an induced subgraph responsible for the MCS, containing $r, r_{i}, r_{j}, r_{k}$ as four black vertices pairwise connectedr, and we can pick three different couples of $r, r_{i}, r_{j}, r_{k}$ such that each couple shares a column (white vertex) that is not shared with the two other of the MCS (see Figure 4, V_4).

Proposition 8 Algorithm Check $V_{-} 4$ is correct and runs in $O\left(m^{3}\right)$ time. 


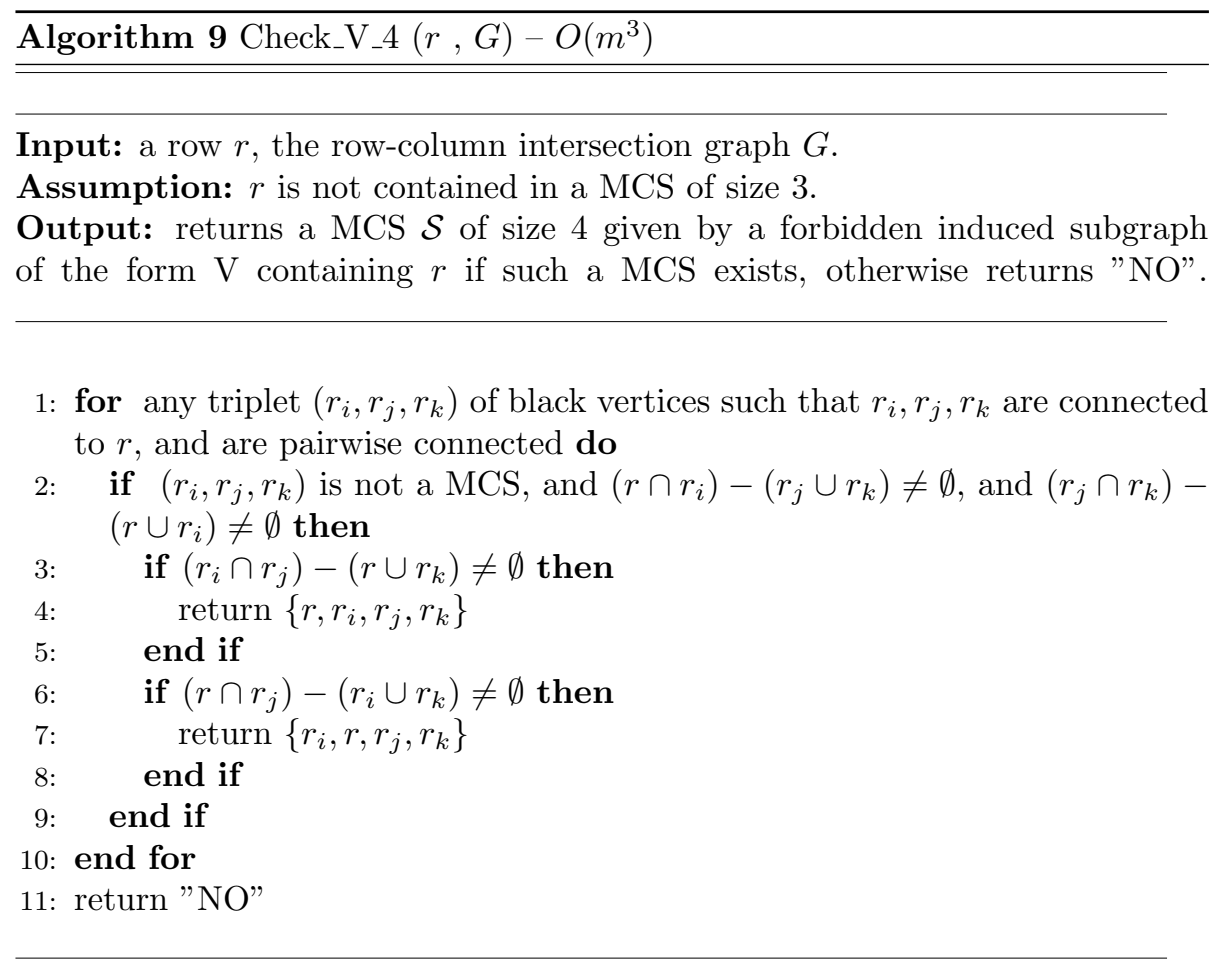


Proof. Algorithm Check_V_4 looks for an induced subgraph with 4 black vertices $\left\{r, r_{i}, r_{j}, r_{k}\right\}$, that are pairwise connected to each other. These 4 black vertices should be such that there exist three different couples of vertices among them, such that two couples are disjoint and the third one (called couple_kernel) overlaps the two first, and the 2 rows of each of these couples share a column that is not shared with the two other rows of the set. In this case, if $\left\{r_{i}, r_{j}, r_{k}\right\}$ is not a MCS, then the subgraph induced by $\left\{r, r_{i}, r_{j}, r_{k}\right\}$ and the 3 columns (white vertices) connected to the 3 couples of rows is of the form $\mathrm{V}$, and is responsible for a MCS $\left\{r, r_{i}, r_{j}, r_{k}\right\}$. Algorithm Check_V_4 looks for two cases, depending on whether $r$ belong to couple_kernel (lines 3-5), or not (lines 6-8).

Next, all the tests performed by Algorithm Check_V_4 (lines 2-9 of the algoritm) on a given triplet $\left(r_{i}, r_{j}, r_{k}\right)$ are achieved in $O(1)$ thanks to the precomputations, and given $r$ there might be $O\left(\mathrm{~m}^{3}\right)$ such triplets. Thus, Algorithm Check_V_4 runs in $O\left(m^{3}\right)$ time.

Next, for a MCS of size 5 , we look for a quadruplet of rows $\left(r_{i}, r_{j}, r_{k}, r_{l}\right)$ such that the set $\left\{r, r_{i}, r_{j}, r_{k}, r_{l}\right\}$ is a MCS of the form V (Figure 4 $\mathrm{V}_{-} 5$ ). Algorithm Check_V_5 looks for an induced subgraph of the form V, consisting of 5 rows (black vertices) $r, r_{i}, r_{j}, r_{k}, r_{l}$ that are pairwise connected, except for a on missing edge, say $\left(r_{a}, r_{b}\right)$ in $\left\{r, r_{i}, r_{j}, r_{k}, r_{l}\right\} \times\left\{r, r_{i}, r_{j}, r_{k}, r_{l}\right\}$, and three columns (white vertices) satisfying the configuration of Figure 4 $V_{-} \_$.

Proposition 9 Algorithm Check_V_5 is correct and runs in $O\left(m^{4}\right)$ time.

Proof. Algorithm Check_V_5 looks for an induced subgraph with 5 black vertices $\left\{r, r_{i}, r_{j}, r_{k}, r_{l}\right\}$, that are pairwise connected, except for one missing edge $\left(r_{a}, r_{b}\right)$ in $\left\{r, r_{i}, r_{j}, r_{k}, r_{l}\right\} \times\left\{r, r_{i}, r_{j}, r_{k}, r_{l}\right\}$. The 4 black vertices that belong to the set with $r$, should correspond to a set of rows that is C1P. Moreover, there should exist two particular rows (black vertices) of the set, with three columns (white vertices) that satisfy the conditions on line 4 of the algorithm in order to fit the configuration depicted in Figure 4 V_5.

Next, all the tests performed by Algorithm Check_V_5 (lines 2-8 of the algoritm) on a given quatruplet $\left(r_{i}, r_{j}, r_{k}, r_{l}\right)$ are achieved in $O(1)$ thanks to the precomputations, and given $r$ there might be $O\left(m^{4}\right)$ such triplets. Thus, Algorithm Check_V_5 runs in $O\left(m^{4}\right)$ time.

\subsubsection{MCS of size larger than 5}

A MCS of the form $\mathrm{V}$ of size larger than 5 contains exactly two kernels. Depending on whether $r$ is a kernel or not, we distinguish two cases.

\section{Case 1: If row $r$ is a kernel of the MCS}

Algorithm Check_V $V_{k}$ recovers a MCS $\mathcal{S}$ of the form $\mathrm{V}$ of size larger than 5 containing $r$ as a kernel, with the assumption that $r$ is not contained in a MCS of size 3, or 4 (Figure $4 . \mathrm{V}_{k}$ ). The principle of the algorithm is similar to Algorithm Check_IV $V_{k}$. It relies in first choosing the second kernel $a$ of the MCS, and the column $c$, of the induced subgraphof type $\mathrm{V}$ responsible for $\mathcal{S}$, 


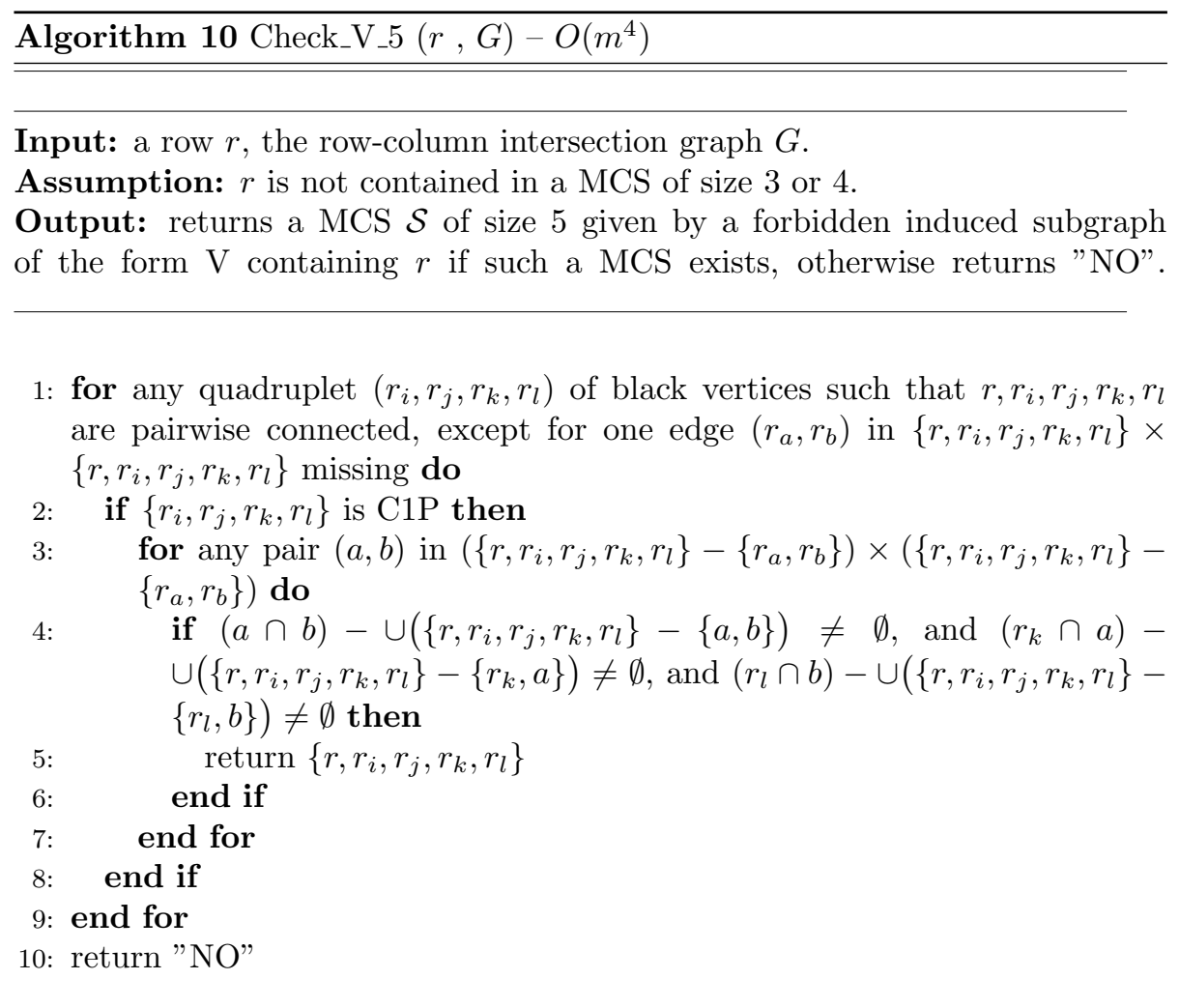


that is contained in both $r$ and $a$, but in no other row of the MCS (see Figure $4 \mathrm{~V}_{k}$ ). Next, it considers the subgraph $H$ of $G$ induced by the set of black vertices (rows) that are neighbors of $r$ and $a$, but do not contain $c$. We denote this subgraph by $H=G[N(r, a)-L(c)]$. Then, it looks for a set of rows $Q$, constituting a chordless path in $H$, such that $\{r\} \cup Q$ is a MCS of the form V.

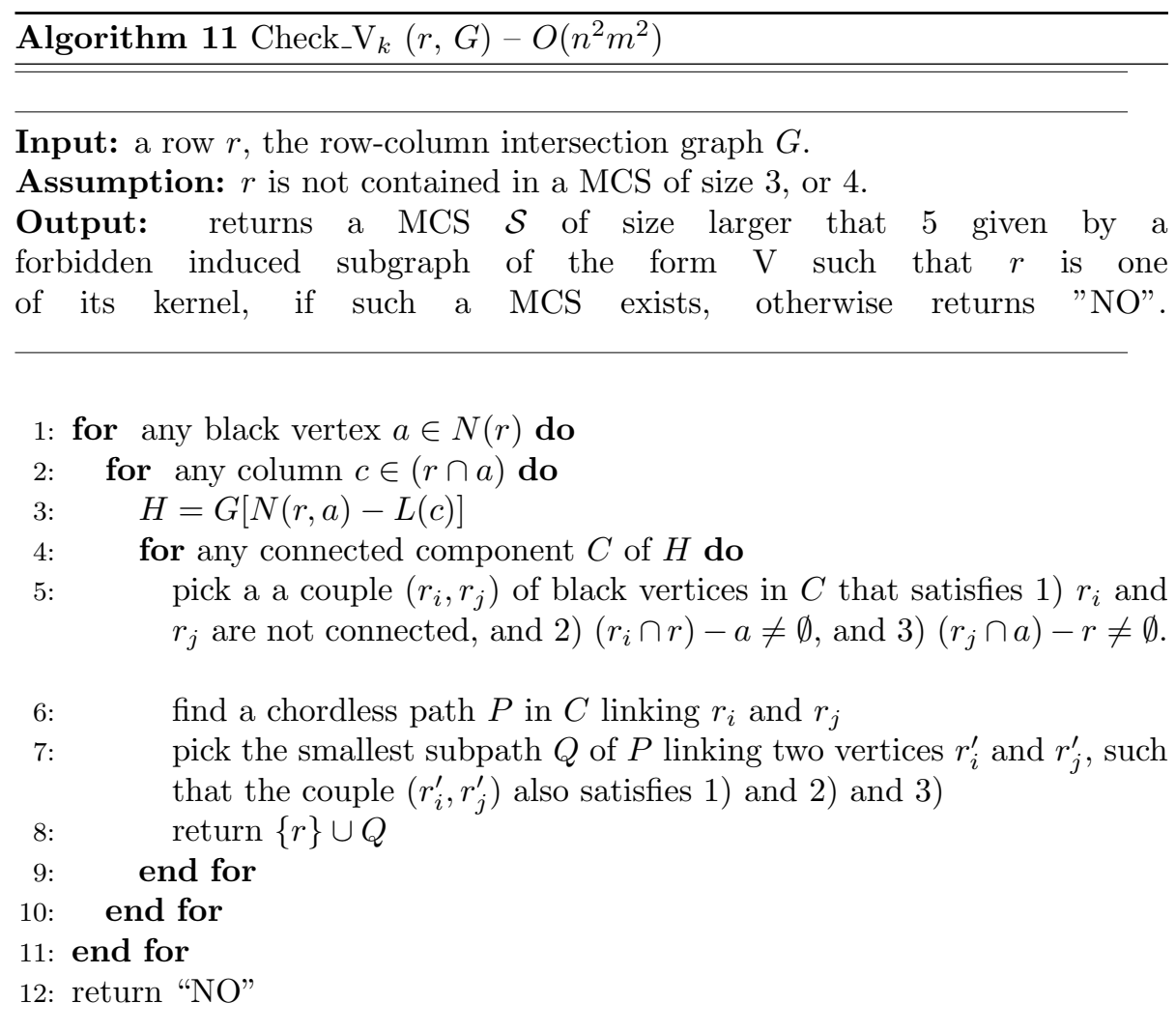

Proposition 10 Algorithm Check- $V_{c}$ is correct and runs in $O\left(n^{2} m^{2}\right)$ time.

Proof. The proofs are similar to the proofs for the correctness and the complexity of Algorithm Check_IV ${ }_{c}$ as the two algorithms are based on the same principle. However, here the complexity is multiplied by a factor $n$ due to considering all black vertices $a \in N(r)$.

Case 2: If row $r$ is not a kernel of the MCS

Algorithm Check_ $\mathrm{V}_{p}$ recovers a MCS S of the form V of size larger than 5 containing $\mathrm{r}$, but not as a kernel, with the assumptions that $r$ is not contained in a MCS of size 3 or 4 , and $r$ does not belong to an induced chordless cycle of $G_{R}$ (Figure $\left.4 \mathrm{~V}\right)$. 
The principle of the algorithm is similar to the principle of Algorithm Check $I V_{p}$. It consists in first choosing the two kernels $(a, b)$ of $\mathrm{S}$ among the black vertices (rows) neighbors of $r$, and the column $c$, of the induced subgraph responsible for $\mathrm{S}$, that is contained in both $a$ and $b$, but in no other row of the MCS. Next, the algorithm calls Algorithm Check_V to look for the MCS S with $r,(a, b), c$, and $G$ given as parameters.

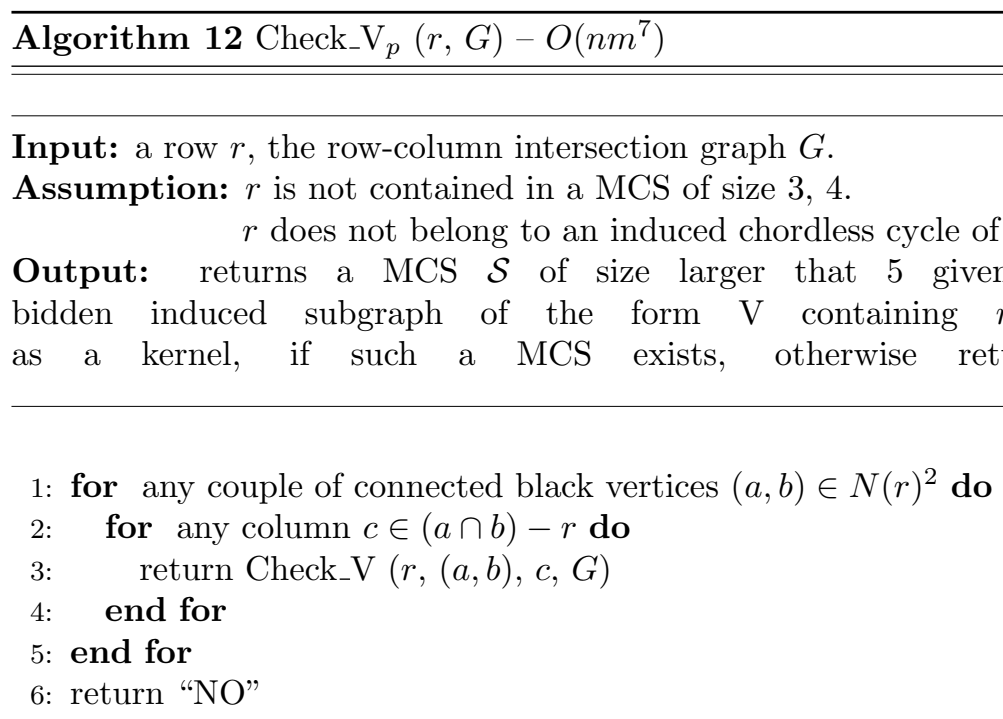

Algorithm Check_V is called in Algorithm Check_V $\mathrm{V}_{p}$. It recovers a MCS $\mathcal{S}$ of the form $\mathrm{V}$ of size larger than 5 containing $r$, given the row $r$, the kernels $a$ and $b$ of the MCS, and the column $c$, of the induced subgraph responsible for $\mathcal{S}$, that is contained in $a$ and $b$, but in no other row of the MCS.

Proposition 11 Algorithm Check $V_{p}$ is correct and runs in $O\left(n m^{7}\right)$ time.

Proof. In order to prove the correctness and the complexity of Algorithm Check $\mathrm{V}_{p}$, we need to prove the correctness and give the complexity of Algorithm Check_V that is called in $\mathrm{Check}_{-} \mathrm{V}_{p}$.

The correctness of Check_V comes from the fact that $r$ does not belong to any chordless cycle in the graph $C$ computed at line 2 of the algorithm by assumption. Let $Q$ be a chordless cycle in the graph $D$ containing vertex $r$, computed at line 9 of the algorithm. Since $r$ does not belong to an induced chordless cycle of the $C$ by assumption, then $Q$ necessarily contains at least one edge belonging to the set $E_{A B} \cup E_{a} \cup E_{b}$.

We first give two trivial but useful properties for the remaining of the proof:

(i) For any two edges of $Q$, there always exists two extremities $u$ and $v$ of these edges, one in each edge, that are not disjoint in the graph $C$, i.e $u \cap v=\emptyset$ 


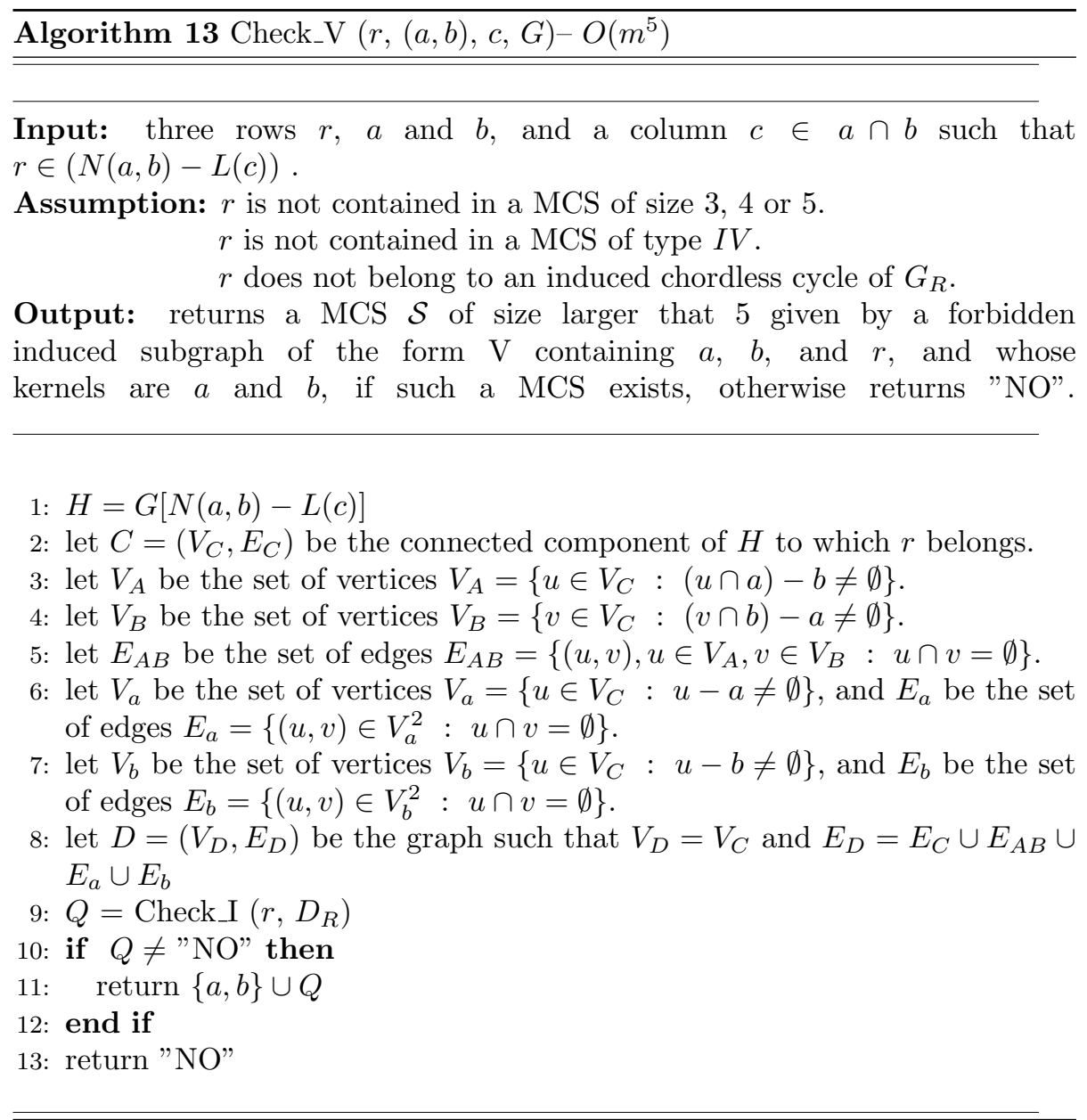


(ii) $V_{A} \subseteq V_{b}$, and $V_{B} \subseteq V_{a}$.

We also prove the following useful property:

(iii) $V_{a} \subseteq\left(V_{B} \cup V_{b}\right)$ and $V_{b} \subseteq\left(V_{A} \cup V_{a}\right)$. Let $x \in V_{a}$, there exists $c$ such that $c \in x$ and $c \notin a$. Then, either $c \notin b$ in which case $x \in V_{b}$, or $c \in b$, which implies that $x \in V_{B}$. The proof is similar for $V_{b} \subseteq\left(V_{A} \cup V_{a}\right)$.

We now prove that the cycle $Q$ necessarily contains at most one edge of the set $E_{A B} \cup E_{a} \cup E_{b}$. Indeed, if $Q$ contains two edges of $E_{A B} \cup E_{a} \cup E_{b}$, let $u, v$ be two disjoint extremities of these edges (Property (i)). We can distinguish 7 cases according to the belonging of $u$ and $v$ to the sets $V_{A}, V_{B}, V_{a}$ and $V_{b}$, and we show in the following that, in all these cases, a chord is induced in the chordless cycle $Q$ in the graph $D$ : contradiction.

1. If $(u, v) \in V_{A}^{2}$ (resp. $\left.(u, v) \in V_{B}^{2}\right)$, then from Property (ii), $(u, v) \in V_{b}^{2}$ (resp. $\left.(u, v) \in V_{a}^{2}\right)$, and thus $(u, v) \in E_{b}$ (resp. $\left.(u, v) \in E_{a}\right)$.

2. If $(u, v) \in V_{a}^{2}\left(\operatorname{resp} .(u, v) \in V_{b}^{2}\right)$, then $(u, v) \in E_{a}\left(\operatorname{resp} .(u, v) \in E_{b}\right)$.

3. If $(u, v) \in V_{A} \times V_{B}$ (or the symmetric), then $(u, v) \in E_{A B}$.

4. If $(u, v) \in V_{A} \times V_{a}$ (or the symmetric), then from Property (iii), $(u, v) \in$ $V_{A} \times V_{B}$ or $(u, v) \in V_{A} \times V_{b}$, and thus $(u, v) \in E_{A B}$ or $(u, v) \in E_{b}$ from cases 3 . and 6 .

5. If $(u, v) \in V_{B} \times V_{b}$ (or the symmetric), then from Property (iii), $(u, v) \in$ $V_{B} \times V_{A}$ or $(u, v) \in V_{B} \times V_{a}$, and thus $(u, v) \in E_{A B}$ or $(u, v) \in E_{a}$ from cases 3 . and 6 .

6. If $(u, v) \in V_{A} \times V_{b}$ (resp. $\left.(u, v) \in V_{B} \times V_{a}\right)$ (or the symmetric), then from Property (ii), $(u, v) \in V_{b}^{2}$ (resp. $\left.(u, v) \in V_{a}^{2}\right)$, and thus $(u, v) \in E_{b}$ (resp. $\left.(u, v) \in E_{a}\right)$.

7. If $(u, v) \in V_{a} \times V_{b}$ (or the symmetric), then from Property (iii), $(u, v) \in$ $V_{b} \times V_{b}$ or $(u, v) \in V_{B} \times V_{b}$, and thus $(u, v) \in E_{b}$ or $(u, v) \in E_{A B} \cup E_{a}$ from cases 1 and 5 .

In consequence, there exits at most one edge, and then exactly one edge of the set $E_{A B} \cup E_{a} \cup E_{b}$ in the cycle $Q$ in the graph $D$. Next, let $\left(r_{i}, r_{j}\right)$ be the only edge of $Q$ belonging to $E_{A B} \cup E_{a} \cup E_{b}$. We show that $\left(r_{i}, r_{j}\right) \notin E_{a} \cup E_{b}$. Indeed, if $\left(r_{i}, r_{j}\right) \in E_{a}$ (resp. $\left.\left(r_{i}, r_{j}\right) \in E_{b}\right)$, then the set $\{a\} \cup Q$ (resp. $\{b\} \cup Q$ ) satisfies the conditions to be a MCS of type IV with $a$ (resp. $b$ ) as kernel, which is impossible by assumption.

So, we have $\left(r_{i}, r_{j}\right) \in E_{A B}-\left(E_{a} \cup E_{b}\right)$. Finally, removing the edge $\left(r_{i}, r_{j}\right)$ from the cycle yields a chordless path $Q$ in $G$ containing $r$ such that each vertex of $Q$ is connected to vertices $a$ and $b$, and the extremities $r_{i}$ and $r_{j}$ of $Q$ satisfy 1) $r_{i}$ and $r_{j}$ are not connected, and 2) $\left(r_{i} \cap a\right)-b \neq \emptyset$, and 3) $\left(r_{j} \cap b\right)-a \neq \emptyset$. and 4) $Q$ does not contain any smaller subpath satisfying conditions 1) and 2) 
and 3). These conditions are necessary and sufficient for the set $\{a, b\} \cup Q$ to form the rows of an induced subgraph of the form $\mathrm{V}$, and this set cannot contain a smaller MCS since such a MCS would be:

- either a MCS of size 3 including $a$ or $b$,

- or a MCS of type II or III necessarily including $a$ or $b$ as kernel,

- or a MCS of type IV and size larger than 3 having $a$ or $b$ as kernel.

- or a MCS of type IV and size larger than 3 having $a$ and $b$ as kernels.

The 3 cases are impossible, since they would induce a chord from the set $E_{A B} \cup$ $E_{a} \cup E_{b}$ in the chordless cycle induced by $Q$ in the graph $D$.

The correctness of Algorithm Check_ $V_{p}$ follows immediately from the correctness of Algorithm Check_V.

Algorithm Check_V calls Algorithm Check_I. Both algorithms have the same time complexity in $O\left(\mathrm{~m}^{5}\right)$ time. It follows immediately that Algorithm Check_IV ${ }_{p}$ runs in $\mathrm{Onm}^{7}$ time.

Acknowledgment We would like to thanks Nicolas Trotignon for his valuable comments on induced subgraphs and also Juraj Stacho for his participation to some meeting on the subject.

\section{References}

[1] A. Bergeron, M. Blanchette, A. Chateau, and C. Chauve. Reconstructing ancestral gene orders using conserved intervals. In Workshop on Algorithms in Bioinformatics (WABI04), number 3240 in LNCS, pages 14-25. Springer, 2004.

[2] G. Blin, R. Rizzi, and S. Vialette. A faster algorithm for finding minimum tucker submatrices. In 6th Conference on Computability in Europe (CiE), volume 6158 of $L N C S$, pages 69-77, 2010.

[3] G. Blin, R. Rizzi, and S. Vialette. A polynomial-time algorithm for finding a minimal conflicting set containing a given row. In $C S R$, volume 6651 of Lecture Notes in Computer Science, pages 373-384. Springer, 2011.

[4] C. Chauve, U.-U Haus, T. Stephen, and V.P. You. Minimal conflicting sets for the consecutive ones property in ancestral genome reconstruction. In Recomb - Comparative Genomics (Recomb-CG09), number 5817 in LNCS, pages 48-58. Springer, 2009.

[5] C. Chauve and E. Tannier. A methodological framework for the reconstruction of contiguous regions of ancestral genomes and its application to mammalian genomes. PLoS Comput Biol, 4(11), 112008. 
[6] M. Dom. Algorithmic aspects of the consecutive-ones property. Bulletin of the Eur. Assoc. for Theor. Comp. Science (EATCS), 98:27-59, 2009.

[7] C.G. Lekkerkerker and J.C. Boland. Representation of a finite graph by a set of intervals on the real line. Fund. Math., 51:45-64, 1962.

[8] T. Nishizeki and Md. S. Rahman. Planar Graph Drawing. World Scientific, 2004.

[9] J. Stoye and R. Wittler. A unified approach for reconstructing ancient gene clusters. IEEE/ACM Trans. Comput. Biol. Bioinf., 6(3):387-400, 2009.

[10] A.C. Tucker. A structure theorem for the consecutive 1s property. Journal of Combinatorial Theory. Series B, 12:153-162, 1972. 